\title{
A Randomized Clinical Trial of Prehabilitation Using Aquatic Exercise in Older Adults with Knee Osteoarthritis Undergoing Total Knee Arthroplasty
}

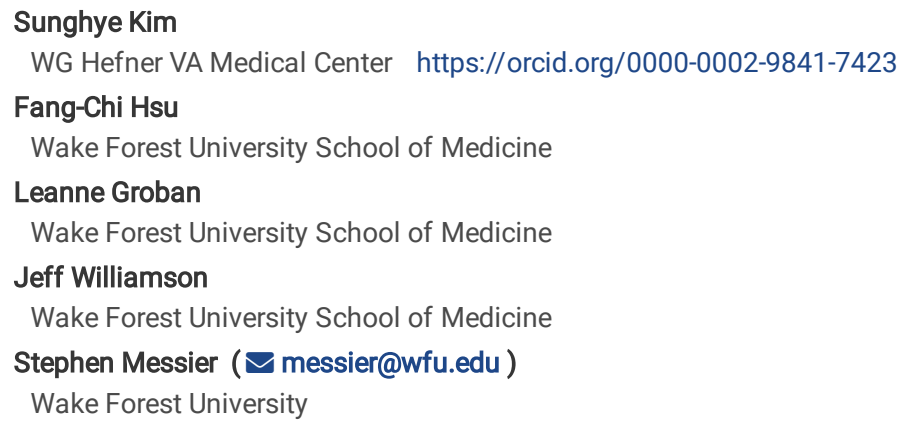




\section{Abstract}

Background: With an aging population, knee osteoarthritis (KOA) is increasingly more prevalent. A significant number of patients with advanced KOA require total knee arthroplasty (TKA). Preoperative physical function predicts postoperative outcomes. We hypothesized that preoperative water exercise to improve preoperative physical function will improve postoperative outcomes after TKA.

Methods: We conducted a randomized controlled trial in 43 participants who were scheduled for TKA and scored at or below 50 th percentile in mobility assessment tool-sf (MAT-sf). All enrolled participants were assessed on 1) clinical osteoarthritis symptom severity using Western Ontario and McMaster Universities Osteoarthritis Index (WOMAC), 2) physical function using Short Physical Performance Battery (SPPB), 3) self-reported mobility using Mobility Assessment Tool-short form (MAT-sf), 4) depression using Geriatric Depression Scale-short form (GDS-sf), 5) cognitive function using Montreal Cognitive Assessment (MoCA). High-sensitivity-C-reactive protein (CRP), tumor necrosis factor-alpha (TNF-a) and interleukin-6 (IL-6) levels were analyzed. Participants were randomly assigned to the aquatic exercise intervention (AEI) or usual care group. The AEI was conducted three times a week for 4-8 weeks, with each session lasting for 60 minutes. Participants in both groups were evaluated within one week before their scheduled surgery, as well as 4 weeks after the surgery. Logistic regressions were used to examine the associations between the intervention and unfavorable outcomes.

Results: The mean age was $67.1( \pm 6.2), 44 \%$ were female, $74 \%$ were White. There is no statistically significant difference in combined outcome of any complication, unscheduled ER visit, and disposition to nursing home or rehab facility by AEI. However, AEI was associated with more favorable functional outcomes including WOMAC scores, chair-stand, MAT-sf, as better mood and cognition and lower blood pressure in multivariable analyses.

Conclusion: 4-8 weeks of aquatic exercise intervention resulted in improved functional outcomes in patients undergoing TKA. A larger study is warranted to explore the role of water exercise in clinical and functional outcomes of TKA.

Trial registration: This clinical trial was registered at ClinicalTrials.gov with ClinicalTrials.gov Identifier of NCT02773745. The date of registration was March 16th 2016. The URL of the trial registry record is https://www.clinicaltrials.gov/ct2/show/NCT02773745?

term=water\&recrs=e\&cond=0steoarthritis\%2C+Knee\&cntry=US\&draw=2\&rank=2.

\section{Background}

According to the U.S. Census Bureau, there were over 49 million people who were 65 and older, accounting for $15 \%$ of the total population in 2016 . It is projected that the population of people $\geq 65$ years old will reach 98 million by the year 2060, representing one in five US residents (1). With an aging population, knee osteoarthritis (KOA) is increasingly more prevalent; it affects $37 \%$ of older adults aged 60 years and older (2). A significant number of patients with advanced KOA require knee arthroplasty and this procedure is expected to increase by $143 \%$ from 2012 to 2050 (3). Although knee arthroplasty is generally successful in limiting pain and improving function, it takes at least 6 months to recover physical function (4) and a significant number of patients experience persistent pain and disability after the procedure (5). Preoperative physical function is known to predict postoperative outcomes (6).

Obesity is a well-known risk factor for KOA. Obesity not only increases biomechanical load to the knee joints, but adipose tissue is known to release a variety of pro-inflammatory factors, such as TNF-a and IL-6 (7). Pain with weight bearing is a universal symptom of KOA. With progression of KOA, pain limits mobility and functionality. This immobility from pain can contribute to weight gain, which in turn enhances further progression of KOA. This cycle is especially true in patients with advanced KOA, such as patients who are considered for a total knee arthroplasty (TKA).

Immobility and weight gain caused by pain can affect postoperative outcomes after TKA in several ways. First, mobility limitation is known to be a risk factor for worse postoperative outcomes; our prospective cohort study discovered that preoperative mobility limitation, assessed by the Mobility Assessment Toolshort form (MAT-sf), is associated with longer postoperative lengths of stay, higher postoperative complication rates, and greater nursing home placement after major surgery (20). Second, elderly patients with limited mobility quickly develop declines in physical function (8) that are well known risk factors for postoperative complications and poor postoperative function (9).

Prehabilitation is defined as enhancing the functional capacity of an individual to enable him/her to withstand an incoming stressor (10). Multiple studies have tested the effect of prehabilitation in patients scheduled for total joint replacement surgery. A systematic review of 35 studies with 2,956 patients reported that prehabilitation was associated with improved postoperative function after total hip arthroplasty (THA) and TKA as well as significantly shorter length of stay after TKA (11). While land exercise is easier and more convenient to perform, it is challenging for patients with knee osteoarthritis since weight bearing can cause pain in patients with advanced KOA. Water exercise can be an alternative to land-based exercise with some evidence that aquatic exercise leads to a short-term improvement in pain and disability in patients with hip or knee osteoarthritis (12).

\section{Methods}

The study was approved by the Institutional Review Board (IRB) of Wake Forest School of Medicine.

\section{Aim}

We designed this pilot clinical trial to test our hypothesis that improving preoperative physical function using aquatic exercise intervention (AEI) in elderly patients with advanced knee osteoarthritis (KOA) who are undergoing TKA will improve postoperative outcomes.

\section{Study Design}

This was a pilot randomized clinical trial. 


\section{Setting}

We enrolled participants from the Preoperative Assessment Clinic at Wake Forest Baptist Medical Center.

\section{Participants}

We recruited participants aged 50 years and older who are scheduled for primary TKA. A trained study coordinator screened potential participants for baseline mobility status, using the mobility assessment tool-short form (MAT-sf). Those participants who score at or below the 50th percentile, based on our prior preoperative study data (20), were considered eligible (e.g. MAT scores $\leq 58$ for men and $\leq 50$ for women). Exclusion criteria included patients who were undergoing knee replacement for indications other than osteoarthritis, revision surgery or bilateral surgery, scheduled for emergency surgery; afraid of water or are not willing to undergo water exercise; having major deficits in hearing or vision; currently exercising more than 3 times a week; participating in another clinical trial; or cannot understand the questionnaires and directions due to cognitive impairment or language barriers. Following these tests, participants underwent a standard medical workup at the discretion of the PAC's attending physician, including American Society of Anesthesiologists (ASA) physical status scoring, and Revised Cardiac Risk Index (RCRI) classification. For each participant, information regarding demographic characteristics, comorbidities, medications were gathered. Written informed consent was obtained from all the participants. At the beginning of the study, we only enrolled participants who are $\geq 65$ years old who's scheduled surgery is more than 8 weeks away. However, due to poor enrollment, the enrollment criteria were changed to participants who are $\geq 50$ years old and have scheduled surgery $\geq 4$ weeks.

\section{Assessment}

Blood pressure, pulse, height and weight were assessed. All enrolled participants were assessed by a trained study coordinator on 1) Western Ontario and McMaster Universities Osteoarthritis Index (WOMAC) pain, stiffness, and physical function (13), 2) physical function using the Short Physical Performance Battery (SPPB), 3) self-reported mobility using Mobility Assessment Tool-short form (MAT-sf) 4) depression using Geriatric Depression Scale Short Form (GDS-sf), and 5) cognitive function using Montreal Cognitive Assessment (MoCA). The WOMAC (score 0-96) is a widely used and extensively validated tool to assess pain, stiffness, and physical function in patients with hip and/or knee osteoarthritis. It has three subscales including pain (5 items, score 0-20), stiffness (2 items, score 0-8) and physical function (17 items, score 0-68) (14). The SPPB consists of repeated chair stands, balance testing, and 4-meter walking speed (total score 0-12). It is known to predict subsequent disability, institutionalization, and mortality (15). The MAT-sf is a 10-item computer-based assessment of mobility using animated video clips (17). The 10 items in the MAT-sf cover a broad range of functioning. The items include walking on level ground, a slow jog, walking outdoors on uneven terrain, walking up a ramp with and without using a handrail, stepping over hurdles, ascending and descending stairs with and without the use of a handrail, and climbing stairs while carrying bags. It has been validated against measures of physical function, including the Pepper Assessment Tool for Disability, the Short Physical Performance Battery, and 400-meter walk test among a population of older community dwellers (17). The GDS-sf (score 0-15) is a 15-item screening tool for depression for older adults and it has been validated in the preoperative setting. Score of $0-5$ is considered as normal, while a score of $>5$ suggests depression (16). The Montreal Cognitive Assessment (MoCA) is a widely validated 30 points test that measures several domains of cognitive function, including short-term memory recall tasks, visuospatial abilities, executive function, attention, concentration, working memory, language, and orientation (18). Baseline blood were drawn at the first visit for high-sensitivity-C-reactive protein (hsCRP), tumor necrosis factor-alpha (TNF-a) and interleukin-6 (IL-6) and, stored at $-80{ }^{\circ} \mathrm{C}$ until the completion of the study and all samples were analyzed together.

Data to calculate Charlson comorbidity (19) was obtained by chart review and the score was calculated.

\section{Intervention Arms}

After baseline assessments, all the enrolled participants were randomly assigned 1:1 to the intervention or control group, using a pre-generated randomization list. The AEI were conducted in warm water (a minimum of 90 degrees) at the Wake Forest Baptist Medical Center Therapy Pool, three times a week, and for 60 minutes each session until the scheduled surgery (4-8 weeks). All the sessions were supervised by an aquatic therapist. The AEI was designed to improve 1) range of motion, 2) muscle strength, and 3) resistance, resulting in improvement in mobility in this low-mobility population. The protocol consists of a warmup (10 minutes); joint range of motion for flexibility and strength (20 minutes); low intensity endurance such as walking to prevent chilling and maintain and/or improve cardiovascular fitness (20 minutes); and end with a cool down (10 minutes). Depending on the participant's tolerance, resistance equipment, such as a noodle and/or neoprene ankle cuff were added to increase the exercise intensity. The participants' exercise log was kept by the aquatic exercise director to record the compliance to the protocol. A trained study coordinator called the intervention group participants weekly to assist with problem solving, reinforce progress, and to encourage adherence. Control group participants received a brochure on perioperative nutrition in addition to the standard cares, such as optimization of their underlying medical problems and perioperative infection prevention measures. The principal investigator who assessed the clinical outcomes, and the two designated trained study coordinator who were not supervising the aquatic exercise and were blinded to the randomization status.

\section{Evaluation before surgery}

Participants in both groups were evaluated one week prior to their scheduled surgery. Blood pressure, pulse, and weight were assessed. Symptoms of osteoarthritis, self-reported mobility, depression, and cognition using the WOMAC, MAT-sf, GDS-sf and MoCA were assessed by a study coordinator. The SPPB was administered by two designated trained study coordinator who were not supervising the aquatic exercise and were masked to the randomization status. At this time, follow up labs were drawn to measure hs-CRP, TNF-a, and IL-6.

\section{Evaluation after surgery}


Participants in both groups were asked to return 4-6 weeks after the scheduled surgery. Blood pressure, pulse, and weight were assessed. Osteoarthritis symptoms, self-reported mobility, depression and cognition, using WOMAC, MAT-sf, GDS-sf and MoCA were assessed by a study coordinator. Physical function was assessed using SPPB by two designated trained study coordinators who were not supervising the aquatic exercise and were masked to the randomization status. Follow up labs were drawn to measure hs-CRP, TNF-a, and IL-6.

\section{Outcome assessment}

The primary outcome of interest was 30 day National Surgical Quality Improvement Project (NSQIP) defined morbidity, including surgical site infection, wound disruption, pneumonia, unplanned intubation, pulmonary embolism, ventilator $>48$ hours, acute renal failure, urinary tract infection, stroke, coma, peripheral nerve injury, cardiac arrest, myocardial infarction, requirement of transfusion, deep vein thrombosis, sepsis and mortality, hospital length of stay, ICU length of stay, delirium, and institutionalization (30). The secondary outcomes were osteoarthritis symptoms using WOMAC, physical function, measured by SPPB, selfreported mobility measured by MAT-sf, depression, measured by GDS, cognition, measured by MoCA, and inflammatory profiles, including CRP, TNF-a, and IL-6.

\section{Statistical analysis}

Due to the novelty of this project, no previous work was available. This study provides preliminary data for estimating effect size of prehabilitation on different outcomes (and corresponding standard deviations) for future larger studies.

All statistical analyses were performed using SAS software, version 9.4 (SAS Institute, Cary, NC). Sample means and standard deviations were computed for the continuous baseline characteristics and counts and proportions were calculated for the discrete baseline characteristics according to intervention groups. For non-normally distributed characteristics, medians, $1^{\text {st }}$ quartile, and $3^{\text {rd }}$ quartile were also calculated. Logistic regressions were used to examine the associations between anesthesia time and unfavorable outcomes, including postoperative complications, longer hospital stay of over 2 days, disposition to nursing home/rehab after adjusting for intervention groups. Odds ratios (ORs) and their $95 \%$ confidence intervals (Cls) were presented. In order to best approximate the conditional normality assumption, the distributions of functional measurements were checked and transformed if needed. Differences in mean values of each functional measurement between intervention groups were estimated using repeated measures analysis of covariance with baseline outcome measure, intervention assignment, visit, and an intervention by visit interaction included in the model. Hypothesis tests for intervention effects at the follow-up visits were performed using contrasts. Overall comparisons between groups across follow-up visits were obtained using a contrast to compare average intervention effects across both follow-up visits. Raw mean and standard deviation were presented at baseline, adjusted least squares means and standard error were presented at post-intervention and post-operative visits.

\section{Results}

We screened 59 patients and randomized 43 participants from 3/17/2016 to 10/31/2017. The enrollment was stopped when we enrolled the number of study participants that we planned. + Figure 1 demonstrates the flow diagram. Table 1 demonstrates the baseline characteristics of the study subjects. There was no difference by the group at baseline.

The median number of water exercise sessions was 12 (IQR = 12 to 16) over a 44 (median 29) day period. The median anesthesia time was 144.5 min (IQR $=129$ to 158 ). After total knee arthroplasty, $13.2 \%$ of patients experienced any complications including superficial, deep and organ space surgical site infection, wound disruption, pneumonia, unplanned intubation, pulmonary embolism, ventilator $>48$ hours, progressive renal insufficiency, acute renal failure, urinary tract infection, stroke, cardiac arrest, myocardial infarction, deep vein thrombosis, return to the operating room, systemic sepsis. The median length of stay was 2 days (IQR=2 from 0 to 2 ) and 2 (5.3\%) patients were discharged to nursing home or rehabilitation facilities. Logistic regression analyses were performed for various unfavorable outcomes, including any complications, unscheduled ER visit, disposition to nursing home/rehab facility, length of stay $>2$ days, or combined outcomes (e.g., any complication, unscheduled ER visit, disposition to nursing home or rehab facility, length of stay> 2 days). Water exercise was not associated with any of the outcomes (Table 2) with a trend for significance for combined outcomes with OR of 0.23 and $p$ value of 0.08 .

Table 3 demonstrates the summary statistics of outcome measurements at baseline, post-intervention and post-operative. Water exercise did not result in statistically significant weight loss, but it was associated lower systolic and diastolic blood pressure at post-operative visit. Water exercise was associated with improved WOMAC score (-11 points) and all three subscales of WOMAC- pain, stiffness and physical function with more significant difference at postintervention visit although the association was attenuated at postoperative visit compared to post-intervention visit. No safety events or unintended effect were observed during the study.

There was no association between water exercise and physical function measured by total SPPB. However, water exercise was associated with improved with chair- stand, one of the subscales of SPPB, especially at post-intervention visit. Water exercise was also associated with improved self-reported mobility, measured by MAT-sf. GDS-sf sores increased in both groups at the post-operative visit (more depression symptoms), although still in normal range. Subjects in the AEl group had less increase in GDS-sf score compared to the control group. Water exercise was also associated with improved cognition, measured by MoCA.

There was no association between AEl and any of the inflammatory markers- (hs-CRP, IL-6, and TNF-a)

To explore the effect of inflammation in mediating the association between water exercise and functional outcomes, we fit two models with and without each inflammatory markers. Other than the effect of CRP's effect on the water exercise and walk speed, there was no mediating effect of any of the inflammatory markers and functional outcomes.

\section{Discussion}


In this pilot clinical trial of 43 participants who were undergoing total knee arthroplasty for advanced osteoarthritis, 12-16 sessions of preoperative water exercise over 4-8 weeks did not result in better postoperative outcomes. However, in logistic regression, water exercise was associated with lower blood pressure, better pain, stiffness, function and total WOMAC scores. Water exercise was also associated with better mobility, mood (lower depression score) and cognition. The association was more prominent at the post-intervention, preoperative period, and the association was generally attenuated after surgery except blood pressure which seems to be only prominent in the post-operative period.

Our hypothesis was that water exercise would improve preoperative physical function that is known to be a predictor of postoperative outcomes. In the elderly population, preoperative frailty, physical function, preoperative exercise capacity, and mobility are reported to be predictive of surgical outcomes ( 9 , 20-24). Prehabilitation using exercise is thought to improve postoperative recovery and physiologic reserve by increasing aerobic capacity and muscle strength (25, 26). The lack of association between water exercise prior to total knee arthroplasty and favorable postoperative outcomes could be explained by our relatively small sample size $(n=43)$. Future larger studies might find an association between preoperative water exercise and improved postoperative outcome. Another possibility is that our intervention in our study might have not been long enough. Prior studies used exercise of one day to eight weeks (11). The AEl group subjects participated in 12 water exercise sessions (IQR = 12 to 16 ) over a 44 (median 29) day period. Although the dose-response relationship between preoperative exercise and postoperative outcomes is not established, a longer duration of intervention might result in more beneficial postoperative outcomes. Another possibility is that our intervention group was at higher risk for poor postoperative outcomes compared to the control group. Although there was no statistical significance, the baseline hs-CRP was numerically higher in the intervention group. Elevated CRP was reported as a predictor of postoperative surgical site infection (27), postoperative infection (28), general complication rate (29). Given our relatively small sample size, we were restricted with the number of co-variates in the model.

Our study found that water exercise resulted in improved osteoarthritis symptoms with decrease in total and each individual WOMAC subcategories of pain, stiffness and function. The total WOMAC score decreased by 11 which is higher than the minimum clinically important difference after total knee arthroplasty (30). This improved WOMAC score continued even in the post-operative period, suggesting that AEI not only improves preoperative but post-operative outcomes of pain, stiffness and function.

In a Cochrane review of 54 randomized controlled trials of land-based exercise for knee osteoarthritis, exercise has shown to reduce pain, improve physical function and quality of life immediately after treatment (31) and the 2019 American College of Rheumatology osteoarthritis guidelines strongly recommended exercise as a treatment for osteoarthritis (32). Interestingly, a study of 126 participants with knee OA and at least one morbidity found that a 20-week individualized, comorbidity adapted land-based exercise program resulted in improvement in WOMAC physical function score of 7.43 (33) while our study of 4-8 weeks of water exercise intervention saw a similar effect (WOMAC physical function score improvement of 7.6) with a much shorter intervention time. Pain associated with land-based exercise can be a significant barrier in exercise among patients with OA (31) while buoyancy provided by water offers less impact on joints and water exercise might be more efficient way to improve knee OA symptoms.

AEI was also associated with chair-stand in SPPB and self-reported mobility, although the association was attenuated after scheduled TKA. It is hypothesized that our study subjects have advanced KOA and the improved symptom and function by our water exercise was not as potent as knee replacement surgery. Future studies of water exercise in less advanced KOA might demonstrate the effect of intervention at earlier stages of KOA can result in delay in the need for arthroplasty.

Water exercise was associated with a better GDS score. While the GDS-sf score increased (more depression symptoms) in the post-intervention and postoperative period in both groups compared to the baseline, the increase in the water exercise group was not as significant. The association between exercise and improved depression was reported before (35), and it is hypothesized that exercise improves depression by increasing neurogenesis and triggering plastic processes (36). However, it is also well known that pain and depression are closely related especially in elderly and common pathologic mechanisms including neuroinflammation is hypothesized to play a role in both conditions (37). Although the increase in GDS-sf in post-intervention (preoperative period) and postoperative period is subclinical, there is a known association between geriatric depression and worse outcomes in many medical conditions and its association with disability (39). Further studies in occurrence of perioperative depression and possible interventions is warranted.

The subjects enrolled in our study had a baseline MOCA score that was close to the mild cognitive impairment cut -off of 26 (18) and water exercise group subjects had improved MOCA scores after intervention. A systematic review with multilevel meta-analysis of 36 studies reported the association between physical exercise and cognitive function (40). However, there are other studies that reported no or only very mild effect of physical exercise on cognitive function $(41,42)$. Our study demonstrated that the MoCA score of the intervention group improved by 1.7 and 2.1 in the post-intervention and post-operative period, while the score did not change in the control group. In a study of 51 healthy middle aged women, 45 minute water exercise sessions twice a week for 6 months period was associated with improved cognition (43). It was hypothesized that exercise intervention, especially aerobic exercise improves cardiorespiratory fitness which in turn improves brain perfusion as well as decreases systemic inflammation and oxidative stress (44). Another possibility is the association between pain and cognition- patients with pain can present with cognitive complaints (45). Water exercise resulted in improved pain and that might explain the improvement in cognition measured by MoCA.

There was no association between inflammatory markers, including hs-CRP, TNF- $a$, and IL-6 levels, and AEI. The baseline hs-CPR was numerically higher in the AEl group, but it was not statistically significant and there was no association between AEl and any of the markers.

Hypertension is a very common condition, especially in the elderly- $63 \%$ of the population aged 60 and over (46). On average, our study subjects had a baseline blood pressure in the pre-hypertensive range. Interestingly, the systolic blood pressure in the intervention group at the post-operative visit decreased by 3.1, while in the control group it increased by 2.4. The blood pressure lowering effect of AEI was significant 4 weeks after surgery, while there was no difference in the post-intervention (preoperative) period. Exercise has been known to be effective in lowering blood pressure (47). This was reported in a clinical trial of water exercise study in participants with resistant hypertension as well- the blood pressure lowering effect persisted 12 weeks after cessation 
of a 12-week training intervention (48). The authors of the report hypothesized that the prolonged effect could be a consequence of immersion in heated water. Nonetheless, it is not clear why the control group subjects in our study experienced an increase in their blood pressure while the intervention group subjects experienced a decrease in blood pressure. Although the change in blood pressure is subclinical, if the trend of decreased blood pressure continues, it can certainly contribute to long-term outcomes.

\section{Limitation}

Our study has a few limitations. First, the sample size is relatively small $(n=43)$ to detect the treatment effect of water exercise. This might be a reason that we did not find any difference in postoperative outcomes in the intervention group vs control group. This small sample size also did not allow us to create multi-variate models with multiple co-variates. Also, we only followed the patients 4 weeks after the surgery. It is known that the functional recovery after TKA occurs over months to a year $(49,50)$, and it is possible that after the 4 weeks follow up the participants' functional outcomes (physical, cognitive, mood) might have different trajectories. Finally, the majority of our patients were white and it might not be generalizable to the general population.

\section{Conclusion}

In this small pilot study of 43 subjects with advanced knee osteoarthritis undergoing total knee replacement surgery, 4-8 weeks of water exercise did not result in improvement in postoperative outcomes. However, the intervention was associated with improved osteoarthritis symptoms, measured by WOMAC. The intervention was also associated with better self-reported mobility, physical function, mood as well as cognition, although the effect tends to be attenuated in the postoperative period. Water exercise was associated with lower systolic and diastolic blood pressure in the postoperative period although there was no effect of the intervention on body weight. Future study of a larger population and less advanced osteoarthritis is warranted to explore the effects of water exercise on osteoarthritis symptoms as well as cognition, depression and blood pressure.

\section{Abbreviations}

\section{MAT-sf}

Mobility Assessment Tool-short form

KOA

Knee Osteoarthritis

TKA

Total Knee Arthroplasty

WOMAC

Western Ontario and McMaster Universities Osteoarthritis Index

SPPB

Short Physical Performance Battery

GDS-sf

Geriatric Depression Scale-short form

MoCA

Montreal Cognitive Assessment

ASA

American Society of Anesthesiologists

RCRI

Revised Cardiac Risk Index

AEI

Aquatic Exercise Intervention

hs-CRP

high-sensitivity-C-reactive protein

TNF-a

Tumor necrosis factor-alpha

NSQIP

National Surgical Quality Improvement Project

IL-6

Interleukin-6

IQR

Interquartile Range

\section{Declarations}

\section{Ethics approval and consent to participate}

The study was approved by the Institutional Review Board (IRB) of Wake Forest School of Medicine and written informed consent was obtained from all the participants. 


\section{Consent for publication}

The authors consent to the publication of this submitted manuscript

\section{Availability of data and materials}

Study data are available upon request

\section{Competing interests}

There is no competing interests by any of the authors

\section{Funding}

This study was funded in part by the Grants for Early Medical/Surgical Specialists' Transition to Aging Research (GEMSSTAR, R03 AG050919) (SK), and the Claude D. Pepper Center Older Americans Independence Center (P30 AG21332) (SK), Wake Forest School of Medicine, Winston-Salem, NC.

\section{Authors' contributions}

Sunghye Kim, Leanne Groban, Jeff Williamson, and Stephen Messier designed this clinical study. Fang-Chi Hus conducted statistical analysis. Sunghye Kim drafted the manuscript. Leanne Groban, Jeff Williamson, and Stephen Messier provided feedbacks and edited the manuscript.

\section{Acknowledgement}

We thank Mr. Peggy Crommer, LRT, CTRS, CPO, the supervisor of recreation therapy at Wake Forest Baptist Health for her selfless dedication to this study. We also thank Ms. Curie Shim for editorial support.

\section{References}

1. Roberts AW, Ogunwole SU, Blakeslee L, Rabe MA. The Population 65 Years and Older in the United States: 2016, American Community Survery Reports2018 Nov 21st, 2019. Available from: https://www.census.gov/content/dam/Census/library/publications/2018/acs/ACS-38.pdf.

2. CDC. Osteoarthritis Atlanta, GA: CDC; 2014 [

3. Inacio MCS, Paxton EW, Graves SE, Namba RS, Nemes S. Projected increase in total knee arthroplasty in the United States - an alternative projection model. Osteoarthritis Cartilage. 2017;25(11):1797-803.

4. Mizner RL, Petterson SC, Snyder-Mackler L. Quadriceps strength and the time course of functional recovery after total knee arthroplasty. J Orthop Sports Phys Ther. 2005;35(7):424-36.

5. Carr AJ, Robertsson O, Graves S, Price AJ, Arden NK, Judge A, et al. Knee replacement. Lancet. 2012;379(9823):1331-40.

6. Fortin PR, Clarke AE, Joseph L, Liang MH, Tanzer M, Ferland D, et al. Outcomes of total hip and knee replacement: preoperative functional status predicts outcomes at six months after surgery. Arthritis Rheum. 1999;42(8):1722-8.

7. Fain JN. Release of interleukins and other inflammatory cytokines by human adipose tissue is enhanced in obesity and primarily due to the nonfat cells. Vitam Horm. 2006;74:443-77.

8. Graf C. Functional decline in hospitalized older adults. Am J Nurs. 2006;106(1):58-67, quiz -8.

9. Makary MA, Segev DL, Pronovost PJ, Syin D, Bandeen-Roche K, Patel P, et al. Frailty as a predictor of surgical outcomes in older patients. J Am Coll Surg. 2010;210(6):901-8.

10. Carli F, Charlebois P, Stein B, Feldman L, Zavorsky G, Kim DJ, et al. Randomized clinical trial of prehabilitation in colorectal surgery. Br J Surg. 2010;97(8):1187-97.

11. Moyer R, Ikert K, Long K, Marsh J. The Value of Preoperative Exercise and Education for Patients Undergoing Total Hip and Knee Arthroplasty: A Systematic Review and Meta-Analysis. JBJS Rev. 2017;5(12):e2.

12. Bartels EM, Lund H, Hagen KB, Dagfinrud H, Christensen R, Danneskiold-Samsoe B. Aquatic exercise for the treatment of knee and hip osteoarthritis. Cochrane Database Syst Rev. 2007(4):CD005523.

13. Bellamy N. Womac Osteoarthritis Index 2012 [Available from: http://www.womac.org/womac/index.htm.

14. Rheumatology ACo. Western Ontario and McMaster Universities Osteoarthritis Index (WOMAC) [Available from: https://www.rheumatology.org/Practice/Clinical/Clinicianresearchers/Outcomes_Instrumentation/Western_Ontario_and_McMaster_Universities_Osteoarth

15. Guralnik JM, Simonsick EM, Ferrucci L, Glynn RJ, Berkman LF, Blazer DG, et al. A short physical performance battery assessing lower extremity function: association with self-reported disability and prediction of mortality and nursing home admission. J Gerontol. 1994;49(2):M85-94.

16. Bass DS, Attix DK, Phillips-Bute B, Monk TG. An efficient screening tool for preoperative depression: the Geriatric Depression Scale-Short Form. Anesth Analg. 2008;106(3):805-9, table of contents.

17. Rejeski WJ, Ip EH, Marsh AP, Barnard RT. Development and validation of a video-animated tool for assessing mobility. J Gerontol A Biol Sci Med Sci. 2010;65(6):664-71.

18. Nasreddine ZS, Phillips NA, Bedirian V, Charbonneau S, Whitehead V, Collin I, et al. The Montreal Cognitive Assessment, MoCA: a brief screening tool for mild cognitive impairment. J Am Geriatr Soc. 2005;53(4):695-9. 
19. Charlson ME, Pompei P, Ales KL, MacKenzie CR. A new method of classifying prognostic comorbidity in longitudinal studies: development and validation. J Chronic Dis. 1987;40(5):373-83.

20. Kim S, Marsh AP, Rustowicz L, Roach C, Leng XI, Kritchevsky SB, et al. Self-reported Mobility in Older Patients Predicts Early Postoperative Outcomes after Elective Noncardiac Surgery. Anesthesiology. 2016;124(4):815-25.

21. Older $\mathrm{P}, \mathrm{Smith} \mathrm{R}$, Courtney $\mathrm{P}$, Hone R. Preoperative evaluation of cardiac failure and ischemia in elderly patients by cardiopulmonary exercise testing. Chest. 1993;104(3):701-4.

22. Lee L, Schwartzman K, Carli F, Zavorsky GS, Li C, Charlebois P, et al. The association of the distance walked in 6 min with pre-operative peak oxygen consumption and complications 1 month after colorectal resection. Anaesthesia. 2013;68(8):811-6.

23. Nutt CL, Russell JC. Use of the pre-operative shuttle walk test to predict morbidity and mortality after elective major colorectal surgery. Anaesthesia. 2012;67(8):839-49.

24. Robinson TN, Wu DS, Sauaia A, Dunn CL, Stevens-Lapsley JE, Moss M, et al. Slower walking speed forecasts increased postoperative morbidity and 1year mortality across surgical specialties. Ann Surg. 2013;258(4):582-8; discussion 8-90.

25. Carli F, Scheede-Bergdahl C. Prehabilitation to enhance perioperative care. Anesthesiol Clin. 2015;33(1):17-33.

26. Kothmann E, Batterham AM, Owen SJ, Turley AJ, Cheesman M, Parry A, et al. Effect of short-term exercise training on aerobic fitness in patients with abdominal aortic aneurysms: a pilot study. Br J Anaesth. 2009;103(4):505-10.

27. Mujagic E, Marti WR, Coslovsky M, Zeindler J, Staubli S, Marti R, et al. The role of preoperative blood parameters to predict the risk of surgical site infection. Am J Surg. 2018;215(4):651-7.

28. Fransen EJ, Maessen JG, Elenbaas TW, van Aarnhem EE, van Dieijen-Visser MP. Enhanced preoperative C-reactive protein plasma levels as a risk factor for postoperative infections after cardiac surgery. Ann Thorac Surg. 1999;67(1):134-8.

29. Gockel I, Dirksen K, Messow CM, Junginger T. Significance of preoperative C-reactive protein as a parameter of the perioperative course and long-term prognosis in squamous cell carcinoma and adenocarcinoma of the oesophagus. World J Gastroenterol. 2006;12(23):3746-50.

30. Clement ND, Bardgett M, Weir D, Holland J, Gerrand C, Deehan DJ. What is the Minimum Clinically Important Difference for the WOMAC Index After TKA? Clin Orthop Relat Res. 2018;476(10):2005-14.

31. Fransen M, McConnell S, Harmer AR, Van der Esch M, Simic M, Bennell KL. Exercise for osteoarthritis of the knee: a Cochrane systematic review. Br J Sports Med. 2015;49(24):1554-7.

32. Kolasinski SL, Neogi T, Hochberg MC, Oatis C, Guyatt G, Block J, et al. 2019 American College of Rheumatology/Arthritis Foundation Guideline for the Management of Osteoarthritis of the Hand, Hip, and Knee. Arthritis Care Res (Hoboken). 2020;72(2):149-62.

33. de Rooij M, van der Leeden M, Cheung J, van der Esch M, Hakkinen A, Haverkamp D, et al. Efficacy of Tailored Exercise Therapy on Physical Functioning in Patients With Knee Osteoarthritis and Comorbidity: A Randomized Controlled Trial. Arthritis Care Res (Hoboken). 2017;69(6):807-16.

34. Taglietti M, Facci LM, Trelha CS, de Melo FC, da Silva DW, Sawczuk G, et al. Effectiveness of aquatic exercises compared to patient-education on health status in individuals with knee osteoarthritis: a randomized controlled trial. Clin Rehabil. 2018;32(6):766-76.

35. Kvam S, Kleppe CL, Nordhus IH, Hovland A. Exercise as a treatment for depression: A meta-analysis. J Affect Disord. 2016;202:67-86.

36. Micheli L, Ceccarelli M, D'Andrea G, Tirone F. Depression and adult neurogenesis: Positive effects of the antidepressant fluoxetine and of physical exercise. Brain Res Bull. 2018;143:181-93.

37. Zis P, Daskalaki A, Bountouni I, Sykioti P, Varrassi G, Paladini A. Depression and chronic pain in the elderly: links and management challenges. Clin Interv Aging. 2017;12:709-20.

38. Ignacio ZM, da Silva RS, Plissari ME, Quevedo J, Reus GZ. Physical Exercise and Neuroinflammation in Major Depressive Disorder. Mol Neurobiol. 2019;56(12):8323-35.

39. Alexopoulos GS. Depression in the elderly. Lancet. 2005;365(9475):1961-70.

40. Northey JM, Cherbuin N, Pumpa KL, Smee DJ, Rattray B. Exercise interventions for cognitive function in adults older than 50: a systematic review with meta-analysis. Br J Sports Med. 2018;52(3):154-60.

41. Htut TZC, Hiengkaew V, Jalayondeja C, Vongsirinavarat M. Effects of physical, virtual reality-based, and brain exercise on physical, cognition, and preference in older persons: a randomized controlled trial. Eur Rev Aging Phys Act. 2018;15:10.

42. Ohman H, Savikko N, Strandberg TE, Kautiainen H, Raivio MM, Laakkonen ML, et al. Effects of Exercise on Cognition: The Finnish Alzheimer Disease Exercise Trial: A Randomized, Controlled Trial. J Am Geriatr Soc. 2016;64(4):731-8.

43. Ayan C, Carvalho P, Varela S, Cancela JM. Effects of Water-Based Exercise Training on the Cognitive Function and Quality of Life of Healthy Adult Women. J Phys Act Health. 2017;14(11):899-904.

44. Hayes SM, Alosco ML, Forman DE. The Effects of Aerobic Exercise on Cognitive and Neural Decline in Aging and Cardiovascular Disease. Curr Geriatr Rep. 2014;3(4):282-90.

45. Moroni C, Laurent B. [Pain and cognition]. Psychol Neuropsychiatr Vieil. 2006;4(1):21-30.

46. Centers for Disease Control and Prevention. Hypertension Prevalence and Control Among Adults: United States, $2015-2016$ 2017 [Available from: https://www.cdc.gov/nchs/products/databriefs/db289.htm.

47. Naci $\mathrm{H}$, loannidis JP. Comparative effectiveness of exercise and drug interventions on mortality outcomes: metaepidemiological study. BMJ. 2013;347:f5577. 
48. Guimaraes GV, Fernandes-Silva MM, Drager LF, de Barros Cruz LG, Castro RE, Ciolac EG, et al. Hypotensive Effect of Heated Water-Based Exercise Persists After 12-Week Cessation of Training in Patients With Resistant Hypertension. Can J Cardiol. 2018;34(12):1641-7.

49. Berghmans DDP, Lenssen AF, Emans PJ, de Bie RA. Functions, disabilities and perceived health in the first year after total knee arthroplasty; a prospective cohort study. BMC Musculoskelet Disord. 2018;19(1):250.

50. Clement ND, Weir D, Holland JP, Gerrand CH, Deehan DJ. An Overview and Predictors of Achieving the Postoperative Ceiling Effect of the WOMAC Score Following Total Knee Arthroplasty. J Arthroplasty. 2019;34(2):273-80.

\section{Tables}

Table 1. Baseline characteristics by intervention groups

\begin{tabular}{llll}
\hline & Intervention group $(\mathrm{n}=20)$ & Standard care group $(\mathrm{n}=23)$ & Overall $(\mathrm{n}=43)$ \\
\hline Age (years) & $67.4 \pm 6.0$ & $66.9 \pm 6.3$ & $67.2 \pm 6.1$ \\
Female (\%) & $10(50 \%)$ & $9(39 \%)$ & $19(44 \%)$ \\
\hline White (\%) & $17(85 \%)$ & $16(70 \%)$ & $33(74 \%)$ \\
\hline Black & $2(10 \%)$ & $7(30 \%)$ & $9(21 \%)$ \\
\hline BMI, mean (kg/m $)$ & $32.9 \pm 7.4$ & $31.9 \pm 5.3$ & $32.4 \pm 6.3$ \\
\hline Charlson's comorbidity score & $3.74 \pm 0.73$ & $3.80 \pm 1.28$ & $3.77 \pm 1.04$ \\
\hline hs-CRP & $5.72 \pm 7.48$ & $2.37 \pm 3.57$ & $3.93 \pm 5.90$ \\
& $2.81(0.63-7.59)^{*}$ & $1.17(0.73-2.46)^{*}$ & $1.97(0.69-3.33)^{*}$ \\
\hline TNF- $\alpha$ & $1.38 \pm 0.58$ & $2.85 \pm 6.43$ & $2.17 \pm 4.73$ \\
& $1.27(0.94-1.55)^{*}$ & $1.26(0.81-1.56)^{*}$ & $1.26(0.85-1.56)^{*}$ \\
\hline IL-6 & $4.16 \pm 4.04$ & $2.68 \pm 2.46$ & $3.37 \pm 3.34$ \\
& $2.84(1.59-5.09)^{*}$ & $2.16(1.40-2.77)^{*}$ & $2.28(1.47-3.94)^{*}$
\end{tabular}

*median (Q1-Q3)

Table 2. Multivariable logistic regression analysis of unfavorable outcomes after controlling for anesthesia time and intervention groups

${ }^{*}$ Combined outcomes= any complication, unscheduled ER visit, disposition to nursing home or home with health care (vs. home without health care)

\begin{tabular}{llll}
\hline & OR & $95 \%$ CI & P value \\
\hline Any complication & 1.5 & $0.21-11.2$ & 0.68 \\
Unscheduled ER visit & 0.13 & $0.01-1.3$ & 0.08 \\
\hline Disposition to nursing home/rehab & 0.43 & $0.04-4.24$ & 0.47 \\
\hline LOS>2 days & 0.17 & 0.02 & 1.8 \\
\hline Combined outcomes $^{*}$ & 0.23 & $0.04-1.17$ & 0.08
\end{tabular}

Table 3. Functional measurements at baseline, post-intervention and post-operative 


\begin{tabular}{|c|c|c|c|c|c|}
\hline & \multirow[t]{2}{*}{ Visits } & Mean $(95 \% \mathrm{CI})^{*}$ & $P$ value for intervention by visit interaction & \multirow[t]{2}{*}{$P$ value for intervention effect at each follow-up visit } & \multirow[t]{2}{*}{ Overall intervention effect (95\% CI), p-value } \\
\hline & & Control & $\mathrm{AEI}$ & & \\
\hline \multirow[t]{3}{*}{ Weight $(\mathrm{kg})$} & Baseline & $92.5 \pm 15.5$ & $93.8 \pm 21.8$ & & $-4.9(-11.9,2.1), 0.16$ \\
\hline & Post-intervention & $94.3 \pm 2.4$ & $89.3 \pm 2.5$ & 0.16 & \\
\hline & Post-operative & $92.3 \pm 2.5$ & $87.0 \pm 2.6$ & 0.16 & \\
\hline \multirow[t]{3}{*}{ SBP } & Baseline & $137.9 \pm 13.6$ & $131.0 \pm 20.5$ & & $-6.3(-15.0,2.4), 0.15$ \\
\hline & Post-intervention & $133.8 \pm 3.5$ & $134.1 \pm 3.6$ & 0.94 & \\
\hline & Post-operative & $141.3 \pm 3.6$ & $127.9 \pm 3.6$ & 0.014 & \\
\hline \multirow[t]{3}{*}{ DBP } & Baseline & $75.3 \pm 10.4$ & $72.0 \pm 10.4$ & & $-2.7(-6.6,1.2), 0.17$ \\
\hline & Post-intervention & $72.9 \pm 1.7$ & $73.9 \pm 1.8$ & 0.69 & \\
\hline & Post-operative & $77.6 \pm 1.8$ & $70.9 \pm 1.8$ & 0.014 & \\
\hline \multirow[t]{3}{*}{ PP } & Baseline & $68.7 \pm 11.0$ & $65.3 \pm 7.7$ & & $-3.6(-7.4,0.2), 0.061$ \\
\hline & Post-intervention & $72.7 \pm 1.5$ & $69.7 \pm 1.6$ & 0.18 & \\
\hline & Post-operative & $78.3 \pm 2.4$ & $73.1 \pm 2.4$ & 0.14 & \\
\hline \multirow[t]{3}{*}{ WOMAC-total } & Baseline & $44.9 \pm 10.7$ & $49.4 \pm 14.9$ & & $-11.1(-15.7,-6.5),<0.001$ \\
\hline & Post-intervention & $48.9 \pm 1.7$ & $37.7 \pm 1.8$ & $<0.001$ & \\
\hline & Post-operative & $39.2 \pm 3.2$ & $28.6 \pm 3.3$ & $<0.026$ & \\
\hline \multirow[t]{3}{*}{ WOMAC-pain } & Baseline & $8.6 \pm 3.0$ & $10.0 \pm 3.7$ & & $-2.0(-3.2,-0.8), 0.002$ \\
\hline & Post-intervention & $9.3 \pm 0.4$ & $7.3 \pm 0.5$ & 0.0029 & \\
\hline & Post-operative & $8.2 \pm 0.7$ & $6.4 \pm 0.7$ & 0.0654 & \\
\hline \multirow[t]{3}{*}{ WOMAC-Stiffness } & Baseline & $4.3 \pm 1.3$ & $4.3 \pm 1.2$ & & $-0.87(-1.36,-0.38), 0.001$ \\
\hline & Post-intervention & $4.5 \pm 0.2$ & $3.3 \pm 0.3$ & 0.0013 & \\
\hline & Post-operative & $4.0 \pm 0.2$ & $3.5 \pm 0.2$ & 0.1417 & \\
\hline \multirow[t]{3}{*}{ WOMAC-function } & Baseline & $32.0 \pm 8.2$ & $35.0 \pm 11.0$ & & $-7.6(-11.6,-3.6),<0.001$ \\
\hline & Post-intervention & $35.0 \pm 1.5$ & $27.6 \pm 1.6$ & 0.0003 & \\
\hline & Post-operative & $26.8 \pm 2.7$ & $18.7 \pm 2.7$ & 0.8764 & \\
\hline \multirow[t]{3}{*}{ SPPB-total } & Baseline & $8.4 \pm 2.1$ & $8.4 \pm 2.3$ & & $0.94(-0.31,2.20), 0.14$ \\
\hline & Post-intervention & $8.7 \pm 0.5$ & $9.5 \pm 0.5$ & 0.18 & \\
\hline & Post-operative & $7.4 \pm 0.5$ & $8.5 \pm 0.6$ & 0.17 & \\
\hline \multirow[t]{3}{*}{ SPPB-balance } & Baseline & $3.6 \pm 0.8$ & $3.6 \pm 0.9$ & & $0.04(-0.49,0.56), 0.88$ \\
\hline & Post-intervention & $3.6 \pm 0.2$ & $3.6 \pm 0.2$ & 0.88 & \\
\hline & Post-operative & $3.6 \pm 0.2$ & $3.8 \pm 0.2$ & 0.55 & \\
\hline \multirow[t]{3}{*}{ SPPB- gait speed } & Baseline & $3.1 \pm 1.0$ & $3.0 \pm 1.0$ & & $0.22(-0.25,0.69), 0.36$ \\
\hline & Post-intervention & $3.1 \pm 0.2$ & $3.4 \pm 0.2$ & 0.37 & \\
\hline & Post-operative & $2.6 \pm 0.2$ & $2.8 \pm 0.2$ & 0.49 & \\
\hline \multirow[t]{3}{*}{ SPPB- chair-stand } & Baseline & $1.7 \pm 1.2$ & $1.9 \pm 1.4$ & & $0.70(0.21,1.19), 0.006$ \\
\hline & Post-intervention & $1.9 \pm 0.2$ & $2.6 \pm 0.2$ & 0.019 & \\
\hline & Post-operative & $1.2 \pm 0.3$ & $1.9 \pm 0.3$ & 0.094 & \\
\hline
\end{tabular}




\begin{tabular}{|c|c|c|c|c|c|c|}
\hline MAT-sf & Baseline & $48.0 \pm 6.6$ & $45.7 \pm 5.8$ & 0.79 & & $4.34(0.90,7.79), 0.015$ \\
\hline & Post-intervention & $44.8 \pm 1.2$ & $49.0 \pm 1.3$ & & 0.019 & \\
\hline & Post-operative & $45.1 \pm 2.0$ & $50.1 \pm 2.0$ & & 0.090 & \\
\hline \multirow[t]{3}{*}{ GDS-sf } & Baseline & $1.7 \pm 1.3$ & $1.9 \pm 2.1$ & 0.35 & & $-1.02(-2.08,0.04), 0.058$ \\
\hline & Post-intervention & $2.9 \pm 0.4$ & $1.7 \pm 0.4$ & & 0.043 & \\
\hline & Post-operative & $2.8 \pm 0.5$ & $2.3 \pm 0.5$ & & 0.51 & \\
\hline \multirow[t]{3}{*}{$\mathrm{MoCA}$} & Baseline & $26.0 \pm 1.8$ & $25.5 \pm 2.2$ & 0.73 & & $1.46(0.46,2.45), 0.005$ \\
\hline & Post-intervention & $25.7 \pm 0.4$ & $27.2 \pm 0.4$ & & 0.008 & \\
\hline & Post-operative & $26.2 \pm 0.4$ & $27.6 \pm 0.4$ & & 0.033 & \\
\hline \multirow[t]{3}{*}{ Log hs-CRP } & Baseline & $0.32 \pm 0.96$ & $0.87 \pm 1.46$ & 0.34 & & $-0.25(-0.69,0.20), 0.27$ \\
\hline & Post-intervention & $0.79 \pm 0.15$ & $0.58 \pm 0.15$ & 0.35 & & \\
\hline & Post-operative & $1.80 \pm 0.28$ & $1.23 \pm 0.29$ & 0.17 & & \\
\hline \multirow[t]{3}{*}{ Log TNF- $\alpha$} & Baseline & $0.33 \pm 0.89$ & $0.25 \pm 0.38$ & 0.88 & & $0.10(-0.07,0.26) 0.25$ \\
\hline & Post-intervention & $0.19 \pm 0.07$ & $0.27 \pm 0.07$ & & 0.39 & \\
\hline & Post-operative & $0.26 \pm 0.07$ & $0.36 \pm 0.07$ & & 0.32 & \\
\hline \multirow[t]{3}{*}{ Log IL-6 } & Baseline & $0.77 \pm 0.61$ & $1.08 \pm 0.84$ & 0.051 & & $-0.02(-0.32,0.28), 0.90$ \\
\hline & Post-intervention & $0.94 \pm 0.11$ & $1.04 \pm 0.11$ & & 0.56 & \\
\hline & Post-operative & $1.53 \pm 0.12$ & $1.31 \pm 0.13$ & & 0.23 & \\
\hline
\end{tabular}

\section{Figures}

Excluded $(\mathrm{n}=16)$

-MAT-sf $>50^{\text {th }}$ percentile $(n=10)$

-Cognitive impairment $(\mathrm{n}=2)$

-Participating in other trial $(\mathrm{n}=1)$

-Withdrawal of consent $(n=2)$

-Depression $(\mathrm{n}=1)$
$\operatorname{AEI}(n=21)$

Post-intervention $(\mathrm{n}=21)$
Control $(\mathrm{n}=22)$

Post-intervention $(\mathrm{n}=22)$

Withdrawal of consent $(n=1)$

Postoperative $(\mathrm{n}=21)$ 
Screening and follow up

\section{Supplementary Files}

This is a list of supplementary files associated with this preprint. Click to download.

- checklist.pdf 